\title{
Cinémas
}

Revue d'études cinématographiques

Journal of Film Studies

\section{Noël Burch, De la beauté des latrines. Pour réhabiliter le sens au cinéma et ailleurs, Paris, L'Harmattan, 2007, 305 p.}

\section{Marie-Hélène Bourcier}

Volume 22, numéro 2-3, printemps 2012

URI : https://id.erudit.org/iderudit/1011660ar

DOI : https://doi.org/10.7202/1011660ar

Aller au sommaire du numéro

Éditeur(s)

Cinémas

ISSN

1181-6945 (imprimé)

1705-6500 (numérique)

Découvrir la revue

Citer ce compte rendu

Bourcier, M.-H. (2012). Compte rendu de [Noël Burch, De la beauté des latrines. Pour réhabiliter le sens au cinéma et ailleurs, Paris, L'Harmattan, 2007, 305 p.] Cinémas, 22(2-3), 181-188. https://doi.org/10.7202/1011660ar d'utilisation que vous pouvez consulter en ligne.

https://apropos.erudit.org/fr/usagers/politique-dutilisation/ 


\section{Noël Burch, De la beauté des latrines. Pour rébabiliter le sens au cinéma et ailleurs, Paris, L'Harmattan, 2007, 305 p.}

À première vue, compte tenu des titres (ironiques?) des deux chapitres qui ouvrent le dernier essai de Noël Burch, "Prolégomènes I» et "Prolégomènes II", on pourrait s'attendre à une hermétique partition de Boulez ou, du moins, à une entrée en matière théorique suivie d'analyses de films qui vaudraient comme autant d'applications de celle-ci: une étude de cas avec L'immortelle (1963) de Robbe-Grillet, un travail sur Hawks et Vidor. L'ouvrage de Burch n'épouse en rien cette facture classique, et ce, à plusieurs niveaux et pour d'excellentes raisons. Sa conception originale constitue en effet un attrait supplémentaire tant elle enrichit la démarche critique qui nous est offerte. Tout d'abord, s'il est vrai que les prolégomènes installent une grille de lecture à la fois complète et subtile du modernisme dans le cinéma français, ils brassent bien plus large. Ils proposent rien de moins qu'une généalogie de la cinéphilie savante française née dans les années 1950, mais aussi - ce qui n'avait jamais été fait et Burch comble là une lacune majeure — du modernisme français dans toutes ses dimensions et sa persistance.

Par ailleurs, d'autres chapitres tout aussi théoriques, comme celui consacré à l'esthétique sadienne française ou, plus exactement, à l'urgence de l'abandonner ("Contre l'esthétique sadienne») et celui consacré à l'ambiguïté des films hollywoodiens de la fin des années 1930 à la fin des années 1950 («Double langage - de l'ambiguïté tendancielle du cinéma hollywoodien») parsèment l'ouvrage. Dans les interstices, on trouvera des chapitres plus longs qui font écho à ces chapitres roboratifs, mais aussi de véritables chroniques engagées en réaction à des films récents qui ne pouvaient laisser insensible un lecteur et un spectateur aussi féministe que Burch. 
De ce point de vue, le chapitre intitulé «Misogynie ordinaire en France: zapping» est sans appel et tristement nécessaire. Il nous renseigne sur la sensibilité critique et politique de Noël Burch, une qualité "féminoïde " pour l'œil moderniste, qui s'exprime clairement dans tout l'ouvrage et le dynamise, faisant de ce livre une véritable autobiographie intellectuelle en mouvement. Mouvement entre deux cultures (Burch fut États-Unien avant de prendre la nationalité française), mais pas seulement, car Burch est un repenti magnifique de ce modernisme qu'il était venu chercher à Paris en 1951 pour s'en défaire par la suite, moyennant une rupture productive. C'est avec un courage et une acuité intellectuelle exceptionnels qu'il raconte et théorise ses investissements et ses désinvestissements tant personnels, sexuels qu'intellectuels, à la lumière de ses engagements politiques de gauche, mais aussi de ses engagements féministes (les deux ne vont pas toujours de pair, a fortiori en France). Ce n'est pas tous les jours que l'on peut lire et comprendre l'itinéraire d'un auteur raconté par lui-même et que marquent un amour invétéré du cinéma motivé par le plaisir et la critique politique et culturelle qu'il procure à tous; ni tous les jours que l'abandon d'une théorie que l'on a pourtant fourbie, comme cette approche hyperformaliste du cinéma que fut Praxis du cinéma paru en 1969, est non seulement assumé, mais débouche sur un rebond critique qui se solde par une analyse inédite et percutante du culte de Sade en liaison avec les transformations du capitalisme, de l'esthétisme, de la politique auteuriste et du mépris de la culture de masse qui s'y rattachent. Cette dimension autocritique particulièrement fructueuse est l'un des ressorts de La beauté des latrines et lui confère à la fois force, honnêteté et agilité intellectuelles.

\section{De l'utilité d'une généalogie du modernisme français pour comprendre la persistance de la cinéphilie savante}

"La première partie de ce livre s'intéresse à trois inventions françaises: le modernisme, le cinéma et la cinéphilie savante» (p. 4). La France s'est enorgueillie d'avoir inventé le cinéma. Il n'est pas sûr qu'en refermant le livre de Noël Burch, le lecteur français puisse concevoir un quelconque sentiment de fierté 
d'avoir inventé avec Louis Delluc le modernisme, la cinéphilie savante et l'auteurisme. Élitiste, abstraite, décontextualisante et dépolitisante, la doctrine moderniste de l'art pour l'art, qui prend sa source, selon Burch, dans la préface de Mademoiselle de Maupin de Théophile Gautier (1835), va faire l'objet de ce qu'il appelle un "détournement moderniste" pour être appliquée au cinéma et permettre d'installer durablement une esthétique de l'art autonome. Selon Burch, le projet de Louis Delluc, à qui l'on doit la création du statut d' " auteur», est :

[...] tout simplement d'annexer pour le modernisme ce nouvel art de masse (le cinéma), de créer un espace de délectation privilégiée par-dessus la tête de son véritable destinataire socioéconomique, le grand public. Et reconfirmer ainsi la supériorité de sa caste. Cette annexion sera accomplie en infusant au cinéma, sur le papier et d'en haut, les deux grands principes du modernisme [...] : l'opacité du signifiant [...] et la dévalorisation des fonctions narratives et référentielles, les films les plus prisés par Delluc le ramenant invariablement aux grands archétypes (p. 12).

Et la stratégie du détournement moderniste ne s'arrête pas là: Delluc s'en resservira pour s'approprier le cinéma hollywoodien de même que l'"Académie française" des Cahiers du cinéma y aura recours pour élire Hitchcock au rang de ses membres privilégiés.

Burch montre comment d'autres caractéristiques du modernisme qui ont marqué les arts en général bien avant le cinéma, vont se retrouver dans la cinéphilie savante française: l'obligatoire séparation de l'art et de la vie, un mode de pensée masculin et masculiniste qui s'accompagne d'un rejet genré de la culture de masse, puisque celle-ci, comme l'a montré Andreas Huyssen, repris par Burch, est codée "féminine», de même que certains de ses genres: le mélodrame, par exemple. La grille d'analyse de Burch fonctionne pour toutes les époques du cinéma français, ce qui témoigne de la persistance du modernisme comme doctrine cinématographique en France encore de nos jours et jusqu'à la Femis, bien après les thèses de Bazin sur l'irréductibilité du réel habité par Dieu, l'esprit de corps des Cahiers du cinéma et l'indispensable célébration de la mise en 
scène comme seul critère d'évaluation d'un "bon" film. Elle permet également une appréciation plus globale de cet exceptionnalisme français par rapport à d'autres pays comme les États-Unis ou l'Angleterre, qui ont su résister à l'emprise moderniste qui irriguait tous les arts (musique, architecture, littérature) et les politiques culturelles du $\mathrm{XX}^{\mathrm{e}}$ siècle. C'est donc un regard à la fois inédit, acéré et nécessaire que porte le critique sur la naissance de la cinéphilie savante française, et notamment sur ses rapports ambigus avec l'extrême droite (en la figure de ce critique de cinéma que fut Rebatet dans Paris-soir et Je suis partout, par exemple), ou encore sur l'anarchisme de droite comme de gauche. Le chapitre intitulé "Cinéma et ressentiment» vient ainsi prolonger les recontextualisations politiques des «Prolégomènes I", tout en offrant une lecture très convaincante qui différencie le cinéma anarchiste droitier d'un Duvivier (le «moi contre tous» de La bandera ou de La belle équipe) et le cinéma anarchiste de gauche d'un Carné/Prévert (le « eux contre nous»). Et pour ce qui est de l'application du formalisme moderniste, Burch passe avec virtuosité de la musique au cinéma, montrant sans grande difficulté les accointances de Robbe-Grillet et du haut modernisme volontiers orientaliste, pour ne pas dire raciste (voir le chapitre intitulé "Retour sur L'immortelle»).

\section{La méthode burchienne}

La grille d'analyse moderniste de Burch est une version augmentée et personnalisée des sept commandements d'Andreas Huyssen. Burch est un pragmatique astucieux et généreux. Il ne manque jamais d'avoir recours aux outils d'analyse qui ont prouvé leur efficacité : ainsi la liste des critères modernistes d'Huyssen, qu'il va largement compléter avec des grilles d'analyse féministes anglo-saxonnes (Moldeski, Williams, Doane et bien d'autres). Il sait reconnaître le mérite de ses rencontres intellectuelles et de la recherche effectuée à quatre mains (nous pensons à sa collaboration avec Geneviève Sellier depuis 1989). Grand passeur, contre vents et marées modernistes, il partage d'ailleurs avec Geneviève Sellier cet engagement volontaire et têtu quand il s'agit d'introduire en France les textes de la critique anglo- 
américaine du cinéma. Non seulement les Cahiers du cinéma, avec lesquels il n'avait pas encore coupé les ponts à l'époque, lui refusent-ils la traduction d'un recueil de textes de la critique états-unienne féministe en 1981, mais ils se fendront d'une critique assassine de celui-ci lors de sa première parution en 1993 (Revoir Hollywood. La nouvelle critique anglo-américaine). Observateur impitoyable des "mœurs de Cinephilia» (p. 67), Burch propose des analyses fines et osées. Il faut un certain culot pour tacler avec autant de force et de rigueur le déferlement misogyne dans le cinéma français des années 1980 et 1990, qu’il s'agisse du cinéma incestueux d'un Brisseau (Noce blanche) ou du cinéma gay d'un Chéreau, d'un Ozon ou encore d'un Honoré, en rappelant à leur bon souvenir l'éphémère cinéma fantastique produit pendant la guerre (Cocteau), et qui perdure un peu après la Libération avec Demy et Guy Gilles. La critique acerbe de la dimension chrétienne de la cinéphilie selon Bazin ne l'empêche pas de préciser l'aspect positif de son projet, qui consiste à dénoncer une certaine cinéphilie et non toute la cinéphilie: "notre plaidoyer n’est donc pas "contre la cinéphilie", ce qui serait absurde, mais plutôt en faveur d'une cinéphilie critique mieux en prise avec la manière dont circule le sens des films dans les sociétés qui les produisent et les regardent» (p. 81).

\section{Un matérialisme culturaliste sensé et bien ancré}

La critique du modernisme et de la cinéphilie savante de Burch débouche donc sur cette réhabilitation du sens évacué par le formalisme moderniste et, par là même, sur la reconstruction d'une analyse exigeante et bien outillée. L'entreprise n'est pas si simple. Il faut jongler avec plusieurs impératifs. Tout d'abord, il s'agit de ne pas jeter l'esthétique avec l'eau du bain moderniste:

[...] si je crois désormais qu’on doit désormais mépriser la question de la beauté ineffable de la mise en scène d'un auteur, il faut essayer de répondre à celle, parfaitement légitime, de la valeur artistique d'une ouvre donnée. Dorénavant, j'entrevois celle-ci comme faite d'une association formellement cohérente et socialement (ethiquement $[s i c])$ signifiante d'éléments esthétiques et non-esthétiques [sic] (au sens de Mukarovsky) et où notamment les contradictions "textuelles" concernent le réel (p. 165). 
Il faut également parer aux travers des lectures culturalistes débridées, où tout se vaut, où tout devient possible et réversible, la logique ultime de ce pluralisme interprétatif étant d'aboutir à la valorisation de lectures individuelles, dépolitisées et décontextualisées. L'autre écueil à éviter est bien sûr la romanticisation de toute lecture oppositionnelle quelle qu'elle soit, et qui fait hâtivement conclure à l'exercice d'une résistance à la domination. Qui ne s'est posé ces questions auxquelles peu de réponses ont été données? C'est tout le mérite de La beauté des latrines d'offrir de réelles solutions à ces interrogations lancinantes de la critique cinématographique culturaliste.

Burch parvient en effet à réconcilier analyse textuelle et ancrage matérialiste de la lecture. Il réussit ni plus ni moins à concevoir une méthode matérialiste à la fois équilibrée et rigoureuse, mais plus souple que celle proposée par Janet Staiger. Staiger recommande en effet de se limiter aux traces matérielles de la réception d'un film pour l'apprécier mais, outre que, comme le fait très justement remarquer Burch, celles-ci sont sélectives et ne sont pas toujours disponibles, il pense qu'il faut aussi tenir compte des ambiguités textuelles et des stratégies des studios à l'égard des divers publics auxquels ils s'adressent: par exemple, le fait que les studios aient conscience que ce sont les femmes qui deviennent prescriptrices des sorties culturelles familiales dans les années 1950 aux États-Unis. L'ambiguïté selon Burch, dérivée avec profit du concept d'ambiguïté défini par William Empson pour la poésie (dans son chapitre intitulé «Double language - De l'ambiguïté tendancielle du cinéma hollywoodien"), devient un outil très efficace appliqué à cette industrie culturelle qu'est le cinéma. Burch va le mettre en œuvre sur des films particuliers (Délivrance, Forfaiture, Taxi Driver), mais l'utiliser également pour analyser certaines périodes cinématographiques: le film noir et le film gothique de la fin des années 1930 à la fin des années 1950, sans oublier ce Hollywood "post-moderne" que Burch ne porte vraiment pas dans son cœur parce qu'il ne ferait selon lui que reproduire un dédain moderniste, "un anti-américanisme chic" finalement non politique destiné à un public averti qui méprise in fine le populaire (Verhoeven, Tarantino, les frères Coen). 
On est loin de «la perception "naïve" des films" (p. 59) modestement revendiquée pour s'éloigner de la geste moderniste et cinéphilique française qu'annonçait Burch dans ses prolégomènes. Avec De la beauté des latrines Burch a réussi son pari :

[...] redéfinir une grille matérialiste de valeurs, à la fois éthiques et artistiques, susceptible de contribuer à l'élaboration, pour le cinéma lisible (au sens de Barthes mais dans une hiérarchie inverse: l'illisible me tombe des mains), d'une poétique impure - en ce qu'il associerait l'esthétique et le non-esthétique, en ce qu'il s'agirait de privilégier autant le discours que sa forme (p. 59).

\section{Faut-il brûler Sade et faire tourner Masoch sans Freud?}

La question peut paraitre étrange mais elle a déjà été posée par Simone de Beauvoir, qui a répondu par la négative, alors que Burch répondrait par la positive, compte tenu de l'icône moderniste française que représente le divin marquis. Mais il fait sans doute mieux ou pire, c'est selon, en critiquant «le haut modernisme dans son incarnation la plus radicale, le dispositif sadien [...] en plaidant pour la réhabilitation d'un dispositif masochien [qu'il] associe à l'économie libidinale de la culture de masse» (p. 78). La réhabilitation de Masoch par Burch, qui vient contrer la consécration élitiste de Sade qui a marqué tout le $\mathrm{XX}^{\mathrm{e}}$ siècle français moderniste, de Tel Quel en passant par Bataille et Klossowski, n'est pas celle de Deleuze, qui à bien des égards n'est pas toujours insensible aux passions modernistes. La réhabilitation burchienne de Masoch est à la fois féministe et culturaliste et peut tout à fait se jouer au cinéma, notamment en ce qui concerne l'analyse des régimes d'identification et de "spectation ${ }^{1}$ " qu'il propose. C'est là toute l'originalité de la thèse du chapitre intitulé "Contre l'esthétique sadienne». Dépassant la mécanique identificatoire cinématographique bloquée de Laura Mulvey, empruntant à l'hypothèse de Baudry qui autoriserait le rapprochement entre le spectateur masculin et une position masochiste masculine, Burch propose une esthétique et une politique féministes (anti-patriarcales) de la position masochiste masculine. Certes, l'admirateur de Masoch est trop subtil pour ne pas déceler l'ambiguiité ou le double langage 
masochiste et percevoir la valeur incertaine de cette place. Néanmoins, sa lecture de certains films de Sternberg avec Marlene Dietrich (Agent X27, Blonde Vénus et L'impératrice rouge) est convaincante: le sous-texte masochiste, voire masochien, est bien là. Donc ça peut marcher. Et sans aucun recours à la psychanalyse ou à la psychologie, car c'est le seul bémol que comportera ce compte rendu d'un livre majeur de la critique cinématographique contemporaine: si je puis me permettre, cher Noël Burch, encore un effort pour culturaliser, contextualiser et resociologiser la psychanalyse et son emprise sur les études cinématographiques. D’ailleurs Freud détestait le cinéma.

Marie-Hélène Bourcier

Université Lille 3

\section{NOTES}

1. «Spectation» est la traduction proposée par Noël Bürch pour le terme anglais spectatorship. 